\title{
Meshless analysis of soil-structure interaction using a MFS-MLPG coupled approach
}

\author{
L. Godinho, P. Amado-Mendes \& A. Tadeu \\ CICC, Department of Civil Engineering, University of Coimbra, Portugal
}

\begin{abstract}
This paper presents a coupling strategy between the Method of Fundamental Solutions (MFS) and the Meshless-Local-Petrov-Galerkin (MLPG) for the analysis of soil-structure interaction problems in the frequency domain. In the proposed approach, the MFS is used to model the outer infinite (or semi-infinite) medium, correctly accounting for the far-field conditions in the soil, while the MLPG is used to simulate the elastodynamic behavior of an embedded solid structure. The MLPG formulation used here is based on the construction of shape functions using the MQ RBF (Multi Quadric Radial Basis Functions); in addition, the test function used to establish the nodal equations is the Heaviside function, leading to the so-called MLPG-5 approach. A direct coupling is considered between the MFS and the MLPG, which implies the construction of a system matrix which accounts for the full coupling effects; in the proposed coupling approach, the possible use of different node distributions along the boundary for the two methods is accounted for, thus rendering the model flexible in allowing different discretization requirements for the soil and for the structure. The algorithm is here applied to 2D problems, and its behavior in terms of convergence and accuracy is analyzed, by comparing its results with those provided by reference solutions. An application example is also presented to illustrate the potential of the proposed approach.
\end{abstract}

Keywords: MFS, MLPG, coupling numerical methods, frequency domain.

\section{Introduction}

When analysing soil-structure interaction and vibration induced by underground transportation systems, the study of wave propagation phenomena in elastic media and the interaction between different solid heterogeneities and the 
elastic hosting media represent important research subjects. In the last decades, the development of a significant number of numerical methods has enhanced our ability to solve more complex and realistic wave propagation and vibration transmission/reduction problems. In these fields, the use of models based on the Finite Difference Method (FDM), Finite Element Method (FEM), Boundary Element Method (BEM), and some variations of these techniques, has been commonly applied and documented, as for instance, in some review works [1, 2].

On the other hand, in the last two decades, a different class of numerical methods, that doesn't present domain or boundary discretization, the so-called meshless methods, has become an interesting alternative to the referred methods, such as, the Method of Fundamental Solutions - MFS and the Method of Particular Solutions - MPS [3, 4], Meshless Local Petrov-Galerkin - MLPG methods [5, 6], and Radial Basis Functions - RBF Collocation methods (e.g., the Kansa's method) [7, 8]. Two truly meshless methods are in the scope of analysis of the present work, namely the MFS and the MLPG. The MFS' solution is found by using a linear combination of fundamental solutions, generated by a set of virtual sources placed outside the analysis domain. The MLPG is a flexible method, in which no elements or meshes are required for field interpolation of the solution variables or for background integration purposes. This method is based on a local weak form and only integration in regularly shaped local subdomains is required, while allowing for the choice of different trial and test functions.

In order to exploit the individual potentialities of distinct numerical methods while minimizing their weaknesses, the development of hybrid numerical schemes has been often suggested by many authors. Although many hybrid algorithms correspond to standard direct coupling methodologies, several iterative coupling procedures between different numerical formulations have recently been proposed, allowing the use of independent discretizations while the corresponding sub-domains can be analysed separately [9, 10]. For dynamic fluid-structure and soil-structure interaction problems, the specific case of coupling the FEM and the BEM has been extensively documented [11, 12]. The coupling of the MFS with other numerical methods has also been proposed, for instance with boundary element formulations [13] and with Kansa's meshless method [14]; in addition, examples of the coupling of the MLPG with FEM and BEM has also been implemented [15].

In this paper, a direct coupling strategy between the MFS and the MLPG is presented in the frequency domain, with the first method modelling the hosting infinite elastic environment while the second one simulates the embedded solid structure. In the implemented coupling strategy, the use of independent point distributions for each part of the model and along the soil-structure interface is allowed, while accounting for a perfect interaction across interfaces. The adopted MLPG formulation is the so-called MLPG-5 approach [6, 16], with the test function used to establish the nodal equations over a local subdomain corresponding to the Heaviside step function. Here, the shape functions are constructed using the MQ (Multi Quadric) RBF. 
The present work is organized as follows: first, the mathematical formulation is presented, with the equations for the elastic problem being described as well as the formulations of the numerical MFS and MLPG schemes. Then, the direct coupling procedure between these techniques is briefly introduced. The model verification comprises the analysis of its convergence and accuracy behaviours in comparison with reference solutions. In the last section, a numerical application example, with a solid tunnel embedded in an elastic formation, is used to illustrate the coupled formulation's applicability in modelling a soil-structure interaction engineering problem.

\section{Mathematical formulation}

In the time domain, the propagation of waves in a homogeneous elastic medium is governed by the vector wave equation (elastic problem)

$$
(\lambda+2 \mu) \nabla \nabla \cdot \mathbf{u}-\mu \nabla \times \nabla \times \mathbf{u}-\rho \frac{\partial^{2} \mathbf{u}}{\partial t^{2}}=0
$$

where $\lambda$ and $\mu$ are Lamé constants, $\rho$ is the density of the elastic medium, and $\mathbf{u}$ is the displacement vector. The application of a time Fourier transformation leads to the following equation, when null initial conditions are assumed:

$$
(\lambda+2 \mu) \nabla \nabla \cdot \mathbf{u}-\mu \nabla \times \nabla \times \mathbf{u}+\omega^{2} \rho \mathbf{u}=0
$$

in which $\omega$ is the angular frequency. The solution of this vector equation can be obtained using different numerical schemes and strategies.

\subsection{Formulation of the MFS}

The MFS is a collocation method which approximates the solution within a given elastic domain as a combination of fundamental solutions generated by a set of virtual sources positioned outside the domain.

Given a set of NS virtual sources, positioned outside the domain (at positions $\mathbf{x}_{0}^{k}$, with $k=1 \ldots N S$ ) so as to avoid singularities, it is possible to write the displacement at point $\mathbf{x}$ along direction $i$ ( $x$ or $y$ ) as:

$$
u_{i}(\mathbf{x}, \omega)=\sum_{k=1}^{N S} G_{i x}\left(\mathbf{x}, \mathbf{x}_{0}^{k}, \omega\right) A_{k, x}+\sum_{k=1}^{N S} G_{i y}\left(\mathbf{x}, \mathbf{x}_{0}^{k}, \omega\right) A_{k, y}
$$

In equation (3), $G_{i x}$ and $G_{i y}$ represent the fundamental solutions which allow calculating the displacement generated at point $\mathbf{x}$ along direction $i$ due to a unit load positioned at $\mathbf{x}_{0}^{k}$ and acting along $x$ or $y$, respectively. In addition, $A_{k, x}$ 
and $A_{k, y}$ represent the a-priori unknown amplitudes of each of the virtual sources. For the case of a homogeneous solid, the fundamental solutions can be defined as [17]:

$$
\begin{aligned}
& G_{x x}\left(\mathbf{x}, \mathbf{x}_{0}^{k}, \omega\right)=\frac{1}{4 \mathrm{i} \rho \omega^{2}}\left[k_{\beta}^{2} H_{0 \beta}-\frac{1}{r} B_{1}+\gamma_{x}^{2} B_{2}\right] \\
& G_{y y}\left(\mathbf{x}, \mathbf{x}_{0}^{k}, \omega\right)=\frac{1}{4 \mathrm{i} \rho \omega^{2}}\left[k_{\beta}^{2} H_{0 \beta}-\frac{1}{r} B_{1}+\gamma_{y}^{2} B_{2}\right] \\
& G_{x y}\left(\mathbf{x}, \mathbf{x}_{0}^{k}, \omega\right)=G_{y x}\left(\mathbf{x}, \mathbf{x}_{0}, \omega\right)=\gamma_{x} \gamma_{y} \frac{1}{4 \mathrm{i} \rho \omega^{2}} B_{2}
\end{aligned}
$$

in which $\quad k_{\alpha}=\omega / c_{d}, \quad k_{\beta}=\omega / c_{s}, \quad \gamma_{i}=\frac{\partial r}{\partial x_{i}}=\frac{x_{i}}{r}(i=1,2)$, $B_{n}=k_{\beta}^{n} H_{n \beta}-k_{\alpha}^{n} H_{n \alpha}, H_{n \beta}=H_{n}^{(2)}\left(k_{\beta} r\right)$ and $H_{n \alpha}=H_{n}^{(2)}\left(k_{\alpha} r\right) ; H_{n}^{(2)}(\ldots)$ is the Hankel function of order $\mathrm{n}$ and of the second kind, while $r=\left\|\mathbf{x}-\mathbf{x}_{0}^{k}\right\|$. Using these equations, strains and stresses at any domain point can be easily obtained making use of classic elasticity equations.

Considering these definitions and imposing the necessary boundary conditions at a set of NS boundary collocation points, positioned along the boundary of the domain, a system of 2 xNS equations on 2 xNS unknowns can then be assembled.

The final system of equations of the MFS can be written as

$$
\left[\begin{array}{l}
\mathbf{H}(\omega) \\
\mathbf{G}(\omega)
\end{array}\right] \mathbf{A}(\omega)=\left[\begin{array}{l}
\overline{\mathbf{t}}(\omega) \\
\overline{\mathbf{u}}(\omega)
\end{array}\right]
$$

in which $\mathbf{G}$ and $\mathbf{H}$ are assembled establishing the essential and the natural boundary conditions, respectively, and $\overline{\mathbf{u}}$ and $\overline{\mathbf{t}}$ incorporate the prescribed displacements and stresses (normal and tangential to the boundary) at the collocation points. The vector of unknowns can then be defined as $\mathbf{A}(\omega)=\left[\begin{array}{lllllll}A_{1, x} & A_{1, y} & A_{2, x} & A_{2, y} & \ldots & A_{N S, x} & A_{N S, y}\end{array}\right]^{T}$.

\subsection{Formulation of the MLPG for closed subdomains}

For the formulation of the MLPG approach used here, a number of nodes is scattered throughout the domain and along its boundary, and the following weak form (obtained considering a Heaviside test function, leading to the so-called MLPG-5 [6]) of the governing equation around node $i$ is considered

$$
-\int_{\Gamma_{s i}} t_{i} \partial \Gamma-\int_{\Gamma_{s u}} t_{i} \partial \Gamma-\int_{\Omega_{i}} \rho \omega^{2} u \partial \Omega=\int_{\Gamma_{s t}} \bar{t}_{i} \partial \Gamma
$$


in which $\Gamma$ is the boundary and $\Omega$ is the domain of analysis for the MLPG, $\Gamma_{s}$ is the boundary of subdomain $\Omega_{i}$ around the node, which can be decomposed in $\Gamma_{s i}$ (internal boundary), $\Gamma_{s u}$ (essential conditions boundary) and $\Gamma_{s t}$ (natural conditions boundary where $\bar{t}_{i}$ is imposed). A schematic representation of the local boundaries around differently located nodes is illustrated in Figure 1.

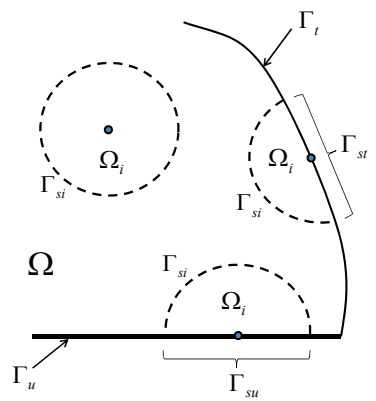

Figure 1: Illustrative representation of a portion of the MLPG analysis domain, identifying the different types of local boundaries.

Using this weak form representation, a system of equations can be defined as

$$
\mathbf{K} \mathbf{u}+\omega^{2} \mathbf{M u}=\mathbf{F}
$$

where $\mathbf{K}$ stands for the stiffness matrix, $\mathbf{M}$ stands for the mass matrix, and $\mathbf{F}$ stands for a vector incorporating the boundary conditions and external loads. The entries of these matrices and vectors can be defined as

$$
\begin{gathered}
K_{i j}=-\int_{\Gamma_{s i}} \mathbf{N D B}_{j} \partial \Gamma-\int_{\Gamma_{\Gamma_{s u}}} \mathbf{N D B}_{j} \partial \Gamma \\
M_{i j}=-\int_{\Omega_{i}} \boldsymbol{\varphi}_{j} \rho \partial \Omega \\
F_{i}=\int_{\Gamma_{s i}} \bar{t}_{i} \partial \Gamma,
\end{gathered}
$$

$\mathbf{N}, \varphi$ and $\mathbf{B}$ being matrices containing the information about the outward pointing normal with respect to the local boundaries, and about shapes functions and their derivatives, respectively; $\mathbf{D}$ is the standard material behaviour (constitutive) matrix, here considered for the case of plane-strain problems and for isotropic elastic materials. Details on these matrices can be found in [16], among other published works. Here, the integration subdomain is considered to be 0.5 times the distance to the nearest neighbour node, while the support domain for interpolation is assumed to be 3.5 times that distance. 
It should be noted, from equation (9), that, if a constant point distribution is used, matrices $\mathbf{K}$ and $\mathbf{M}$ only need to be computed once, even when several frequencies are to be analysed.

The shape functions adopted in this work are built making use of the MQ $\mathrm{RBF}$, incorporating polynomial terms of the first order. Considering a point $\mathbf{x}^{i}$, and defining an interpolation domain around the point including $\mathrm{M}$ distinct nodes, the displacement along direction $k$ can be defined as

$$
u_{k}^{i}=\sum_{j=1}^{M} R^{j}\left(\mathbf{x}^{i}\right) \times B_{k}^{j}+\sum_{j=1}^{N P} P^{j}\left(\mathbf{x}^{i}\right) \times C_{k}^{j}
$$

with the following constraint

$$
\sum_{j=1}^{M} P^{i}\left(\mathbf{x}^{j}\right) \times B_{k}^{j}=0, \text { for } i=1 . . N P
$$

where $N P$ equals 3 when the polynomial terms are of first order, in that case $P^{j}$ being the $j^{\text {th }}$ element of $\mathbf{P}(x, y)=\left[\begin{array}{lll}1 & x & y\end{array}\right] ; R^{j}\left(\mathbf{x}^{i}\right)=\left(r^{2}+c^{2}\right)^{\beta} \quad(\beta=1.03$ is adopted here, following [16]), with $r=\left\|\mathbf{x}^{i}-\mathbf{x}^{j}\right\|$ and $c$ being a free parameter. Writing equations (13) and (14) for a set of $\mathrm{M}$ nodes within a local interpolation domain, the system $\mathbf{u}_{k}=\mathbf{R}_{0} \mathbf{Q}_{k} \Leftrightarrow \mathbf{Q}_{k}=\mathbf{R}_{0}{ }^{-1} \mathbf{u}_{k}$ can be defined ( $\mathbf{u}_{k}$ being the vector containing the displacements at the nodal points of the interpolation domain, and $\mathbf{Q}_{k}=\left[\begin{array}{llllll}B_{k}^{1} & \ldots & B_{k}^{M} & C_{k}^{1} \ldots C_{k}^{N P}\end{array}\right]^{T}$ ), and the displacement at a generic point $\mathbf{x}$, not coinciding with a node, can be written as

$$
u_{k}(\mathbf{x})=\mathbf{R}^{T}(\mathbf{x}) \mathbf{R}_{0}^{-1} \mathbf{u}_{k}=\boldsymbol{\Phi}(\mathbf{x}) \mathbf{u}_{k}
$$

with $\boldsymbol{\Phi}(\mathbf{x})$ being the so-called shape function at $\mathbf{x}$, and $\mathbf{R}=\left[R^{1}(\mathbf{x}) \ldots R^{M}(\mathbf{x}) P^{1}(\mathbf{x}) \ldots P^{N P}(\mathbf{x})\right]^{T}$ being a vector built using the coefficients from equation (13), containing the values of the RBFs and polynomial functions at point $\mathbf{x}$. It should be noted that, in practice, $\boldsymbol{\Phi}(\mathbf{x})$ contains nothing more than interpolation coefficients that allow computing the displacement at point $\mathbf{x}$ from the displacements at the nodal points used for interpolation.

\section{MFS-MLPG coupling}

The MFS and the MLPG parts of the model are coupled using a direct approach. The coupling strategy is, however, developed so that different discretizations can be used for each part of the model (e.g. for each numerical model). For that purpose, consider a portion of the interface connecting the MFS and the MLPG 
subdomains as illustrated in Figure 2, along which equilibrium and continuity conditions must be enforced.

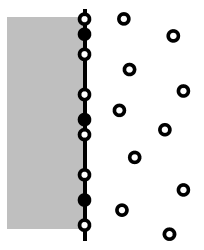

- MLPG node

- MFS collocation

point

Figure 2: $\quad$ MFS-MLPG interface.

To enforce displacement continuity, the corresponding continuity equations must be written for each of the collocation points on the MFS part of the model. Considering a collocation point $\mathrm{N}$, coinciding with a MFS node, the corresponding continuity equations can be written just as

$$
u_{k, M F S}\left(\mathbf{x}^{N}, \omega\right)-u_{k, M L P G}\left(\mathbf{x}^{N}, \omega\right)=0
$$

where the subscripts MFS or MLPG indicate which part of the model is to be considered. To calculate each term, equations (3) and (15) must be used respectively for the MFS and for the MLPG.

The traction equilibrium conditions are, in turn, established at the MLPG boundary nodes, and are thus considered as additional terms in the MLPG equations established at those nodes. Considering equation (9), and analysing the MLPG nodes along the interface with the MFS domain, one may then write at node NM:

$$
\mathbf{F}_{M L P G}\left(\mathbf{x}^{N M}, \omega\right)+\mathbf{F}_{M F S}\left(\mathbf{x}^{N M}, \omega\right)=0
$$

in which $\mathbf{F}_{M L P G}\left(\mathbf{x}^{N M}, \omega\right)$ is obtained from equation (12) and $\mathbf{F}_{M F S}\left(\mathbf{x}^{N M}, \omega\right)$ is the vector of tractions generated by the MFS part of the domain at the analysed node $\mathrm{NM}$; the direction of the outward pointing normal is adopted for each subdomain. To compute these tractions, integration of the corresponding stresses along the boundary must be performed.

\section{Model verification}

To verify the proposed coupled model, consider an infinite medium in which a circular solid inclusion of unit radius is located, centered at the origin, excited by a dynamic load acting at some point of the host elastic medium. For this case, if the same elastic properties are ascribed both to the host medium and to the inclusion, the solution of the problem can be defined analytically using equations (4)-(6), depending on the direction of the load. Thus, to verify the proposed model, a test problem is considered assuming that the load acts along the vertical 
direction and that it is positioned at point $\mathbf{x}_{0}$ with coordinates $(-10.0 \mathrm{~m} ; 2.0 \mathrm{~m})$, and that the elastic properties of the host solid and of the inclusion materials are $E=500 \mathrm{MPa}, v=0.2$ and $\rho=2000 \mathrm{~kg} / \mathrm{m}^{3}$. The response of this system is then calculated for two specific frequencies of $100 \mathrm{~Hz}$ and $500 \mathrm{~Hz}$, over a set of points located within the inclusion. For the numerical model, fixed numbers of collocation points are defined for the MFS ( 10 for $\mathrm{f}=100 \mathrm{~Hz}$ and 20 for $\mathrm{f}=500 \mathrm{~Hz}$ ), and the number of boundary nodes of the MLPG is then progressively increased; for the MFS, the sources are positioned at a distance of $0.5 \mathrm{~m}$ from the interface. Figure 3a illustrates a sample point distribution for this simple test problem, while Figures $3 b$ and $3 c$ exhibit the convergence of the method (using the relative L2 error norm) for the two frequencies. To determine the relative error, the following expression is used:

$$
e=\sqrt{\sum_{i}^{N M L P G}\left(u_{\text {exact }}^{i}-u_{\text {numerical }}^{i}\right)^{2} / \sum_{i}^{N M L P G}\left(u_{\text {exact }}^{i}\right)^{2}}
$$

for which the displacements are evaluated at all MLPG nodes. In that expression, NMLPG is the number of nodes in the MLPG domain.

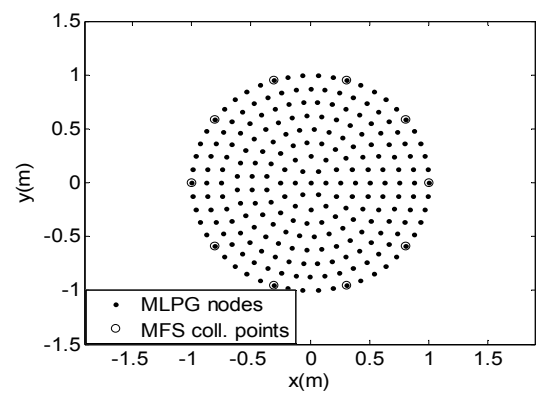

a)

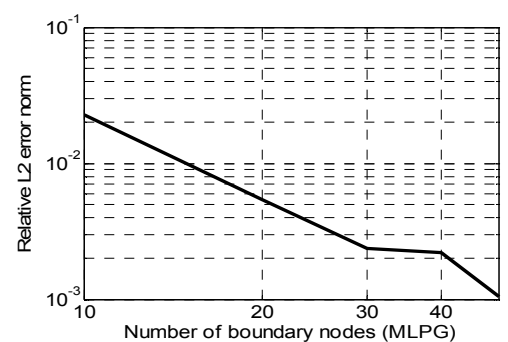

b)

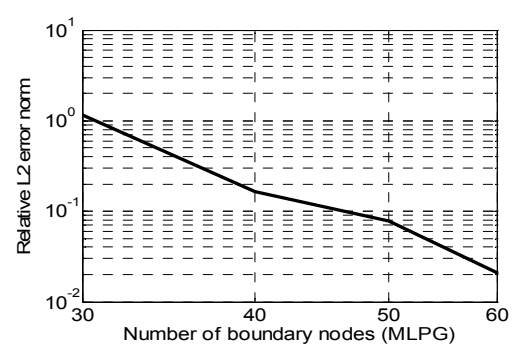

c)

Figure 3: Verification of the MFS-MLPG model: a) sample point distribution for the test problem; b) convergence of the response for $\mathrm{f}=100 \mathrm{~Hz}$; c) convergence of the response for $\mathrm{f}=500 \mathrm{~Hz}$. 
Observing the two curves plotted in Figure 3, it becomes clear that the error is progressively reduced as the number of points in the MLPG part of the model is increased, indicating that the response is converging to the solution of the problem. These results also indicate that, for this situation, the MLPG is limiting the accuracy of the computed results, and that only a very small number of MFS points is required to attain good results. This fact is relevant since it further confirms the importance of allowing independent discretizations to be used for each model.

To further verify the coupled model, an additional test was performed, considering the properties of the host medium to be different from those inside the circular inclusion. For this purpose, the properties of the elastic material of the inclusion are assumed to be $E_{2}=2 \mathrm{GPa}, v_{2}=0.2$ and $\rho_{2}=2500 \mathrm{~kg} / \mathrm{m}^{3}$. A reference solution for this case was obtained making use of the Boundary Element Method (BEM), discretizing the interface between solids using 100 boundary elements with constant interpolation function. Figure 4 illustrates the computed displacements at a receiver located at $\mathbf{x}_{R}$ with coordinates $(2.0 \mathrm{~m} ; 2.0 \mathrm{~m})$ for frequencies between $2 \mathrm{~Hz}$ and $200 \mathrm{~Hz}$, when 20 collocation nodes are used for the MFS, and 50 boundary nodes are used for the MLPG. As can be observed in the presented plots, an excellent agreement was found between the BEM and the proposed coupled approach.

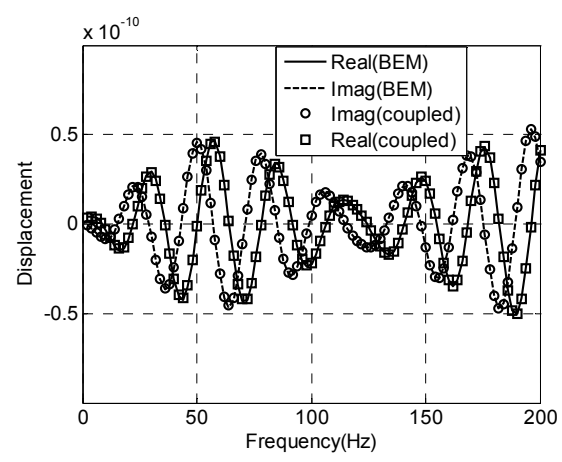

a)

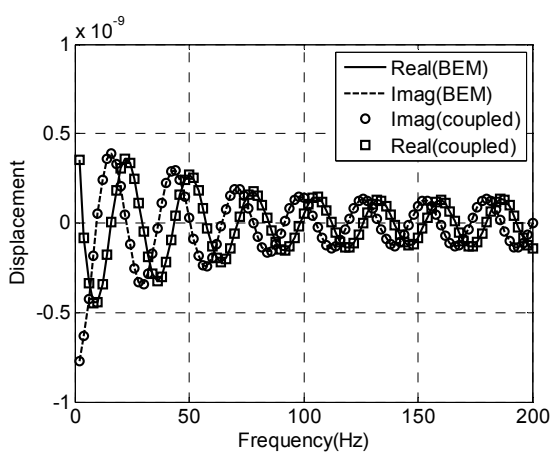

b)

Figure 4: Responses computed using the MFS-MLPG model and a BEM reference model at a single receiver, along a full frequency range: a) horizontal $(x)$ displacement; b) vertical ( $y$ ) displacement.

\section{Numerical application example}

As an application example of the proposed methodology, consider the case of a tunnel with the geometry depicted in Figure 5, with an external radius of $3.0 \mathrm{~m}$. The structure of the tunnel is assumed to be homogeneous, with properties $E=29 \mathrm{GPa}, \quad v=0.2$ and $\rho=2500 \mathrm{~kg} / \mathrm{m}^{3}$, while the surrounding soil is characterized by $E=500 \mathrm{MPa}, v=0.2$ and $\rho=1800 \mathrm{~kg} / \mathrm{m}^{3}$. The model used 


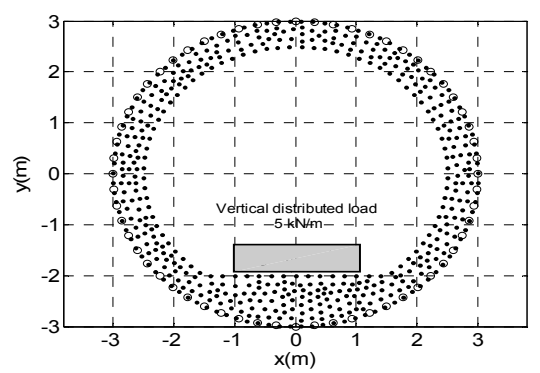

Figure 5: $\quad$ Model of the buried tunnel excited by a distributed dynamic load.

for this problem incorporates 60 collocation points for the MFS, and a total of 488 points for the MLPG, as illustrated also in Figure 5.

Time responses have been calculated for this problem, assuming that the excitation load (a vertical distributed load) has a temporal variation defined by a Ricker wavelet, with a central frequency of $100 \mathrm{~Hz}$. Responses are first calculated in the frequency domain for frequencies between $2 \mathrm{~Hz}$ and $256 \mathrm{~Hz}$, considering complex frequencies with a small imaginary part $\left(\omega_{c}=2 \pi f-0.7 \times \mathrm{i} \times 2 \pi \Delta f\right)$, to avoid aliasing phenomena [18]. Figure 6 exhibits the response computed throughout the surrounding soil (along a grid of receivers placed between $x=-20 \mathrm{~m}$ and $x=20 \mathrm{~m}$, and between $y=-15 \mathrm{~m}$ and $y=15 \mathrm{~m})$, at two time instants $(\mathrm{t}=30.3 \mathrm{~ms}$ and $\mathrm{t}=50.3 \mathrm{~ms})$, in terms of horizontal and vertical displacements.
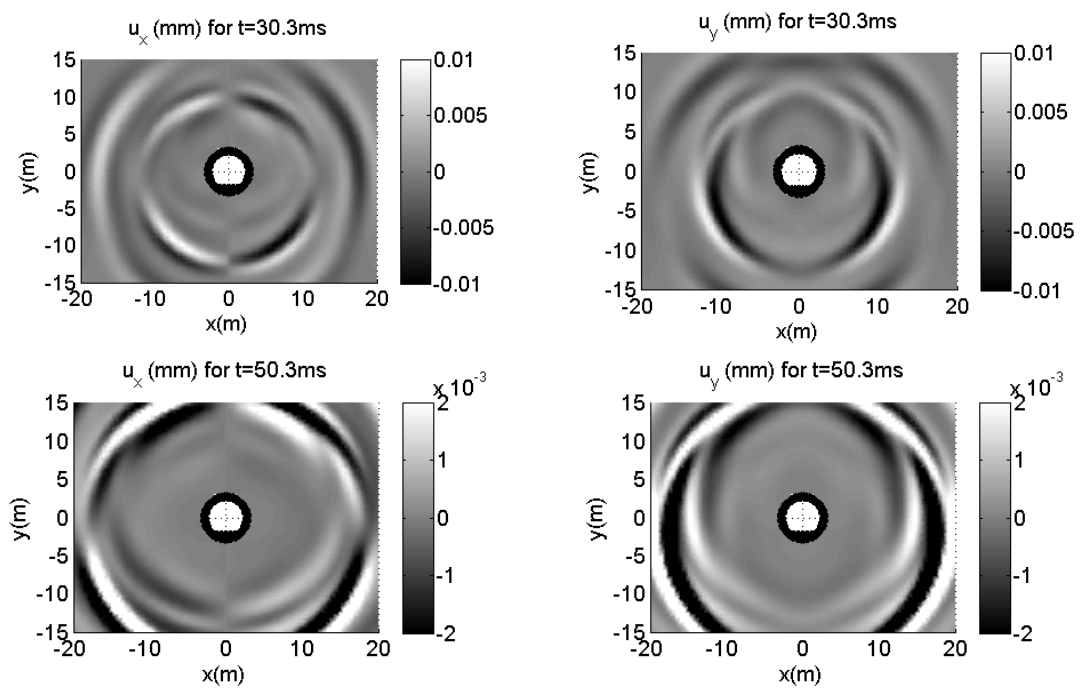

a)

Figure 6: Horizontal (left) and vertical (right) displacements at time instants of $30.3 \mathrm{~ms}$ (a) and $50.3 \mathrm{~ms}$ (b). 
At the earlier time, the displacements in Figure 6a) clearly reveal two different wavefronts radiating from the tunnel structure, corresponding mostly to $\mathrm{P}$ waves and $\mathrm{S}$ waves traveling in the soil with velocities of $555.5 \mathrm{~m} / \mathrm{s}$ and $340.2 \mathrm{~m} / \mathrm{s}$. Additional wavefronts are also visible which are probably related to second order pulses originated by the vibrating tunnel structure; these waves exhibit, however, lower amplitudes. At the later time, these waves have propagated away from the structure, and very little energy is visible at regions closer to the tunnel.

\section{Final remarks}

A numerical model coupling the MFS and the MLPG5 (with Heaviside test functions) has been presented in this work for the analysis of coupled soilstructure interaction. A direct coupling between the two methods is performed, leading to a fully meshless model which allows independent point distributions for the MFS and MLPG subdomains. The model was verified against a simple analytical solution, for which convergence to the correct solution was observed when denser point distributions were used. Additionally, good results were registered when comparing the results provided by the model with reference BEM results, for the dynamic response of a buried circular elastic inclusion.

\section{Acknowledgements}

The financial support by FCT (Fundação para a Ciência e a Tecnologia) and COMPETE, through project PTDC/ECM/114505/2009, is greatly acknowledged.

\section{References}

[1] Yang, Y.B. and Hsu, L.C., A review of researches on ground-borne vibrations due to moving trains via underground tunnels. Advances in Structural Engineering, 9(3), pp. 377-392, 2006.

[2] Clouteau, D., Cottereau, R. and Lombaert, G., Dynamics of structures coupled with elastic media - A review of numerical models and methods. Journal of Sound and Vibration, 332, pp. 2415-2436, 2013.

[3] Golberg, M.A. and Chen, C.S., The method of fundamental solutions for potential, Helmholtz and diffusion problems, in Boundary Integral Methods: Numerical and Mathematical Aspects, ed. M.A. Golberg, WIT Press and Computational Mechanics Publications, Boston, Southampton, pp. 103-176, 1999.

[4] Li, Z.-C., Young, L.-J., Huang, H.-T., Liu, Y.-P. and Cheng, A. H.-D., Comparisons of fundamental solutions and particular solutions for Trefftz methods. Engineering Analysis with Boundary Elements, 34, pp. 248-258, 2010 . 
[5] Atluri, S.N. and Zhu, T., A new meshless local Petrov-Galerkin (MLPG) approach in computational mechanics. Comput. Mech., 22, pp. 117-127, 1998.

[6] Atluri, S.N., The Meshless Method (MLPG) for Domain and BIE Discretizations, Tech Science Press, Encino, CA, 2004.

[7] Kansa, E., Multiquadrics - A scattered data approximation scheme with applications to computational fluid-dynamics. I Surface approximations and partial derivative estimates. Comput. Math. Appl., 19, pp. 127-145, 1990.

[8] Kansa E., Multiquadrics - A scattered data approximation scheme with applications to computational fluid-dynamics. II Solutions to parabolic, hyperbolic and elliptic partial differential equations. Comput. Math. Appl., 19, pp. 147-161, 1990.

[9] Soares Jr., D., von Estorff, O. and Mansur, W.J., Efficient nonlinear solidfluid interaction analysis by an iterative BEM/FEM coupling. International Journal for Numerical Methods in Engineering, 64, pp. 1416-1431, 2005.

[10] Soares Jr., D. and Godinho, L., An optimized BEM-FEM iterative coupling algorithm for acoustic-elastodynamic interaction analyses in the frequency domain. Computers and Structures, 106-107, pp. 68-80, 2012.

[11] Savin, E. and Clouteau, D., Elastic wave propagation in a 3-D unbounded random heterogeneous medium coupled with a bounded medium. Application to seismic soil-structure interaction (SSSI), International Journal for Numerical Methods in Engineering, 54, pp. 607-630, 2002.

[12] Adam, M. and von Estorff, O., Reduction of train-induced building vibrations by using open and filled trenches. Computers and Structures, 83, pp. 11-24, 2005.

[13] Castro, I. and Tadeu, A., Coupling the BEM/TBEM and the MFS for the numerical simulation of elastic wave propagation. Engineering Analysis with Boundary Elements, 36, pp. 169-180, 2012.

[14] Godinho, L. and Soares Jr., D., Frequency domain analysis of fluid-solid interaction problems by means of iteratively coupled meshless approaches. CMES - Computer Modeling in Engineering and Sciences, 87(4), pp. 327354, 2012.

[15] Liu, G.R. and Gu, Y.T., Meshless local Petrov-Galerkin (MLPG) method in combination with finite element and boundary element approaches. Comput. Mech., 26, pp. 536-546, 2000.

[16] Xiao, J. and McCarthy, M., A local Heaviside weighted meshless method for two-dimensional solids using radial basis functions. Computational Mechanics, 31, pp. 301-315, 2003.

[17] Tadeu, A.J.B. and Kausel, E., Green's functions for two-and-a-half dimensional elastodynamic problems. Journal of Engineering Mechanics ASCE, 126, pp. 1093-1097, 2000.

[18] Kausel, E. and Roesset, J., Frequency domain analysis of undamped systems. Journal of Engineering Mechanics ASCE, 118(4), pp. 721-734, 1992. 